\title{
THE UNIQUENESS OF THE SOLUTION OF THE SCHRÖDINGER EQUATION WITH DISCONTINUOUS COEFFICIENTS
}

\author{
Willi JäGeR ${ }^{1}$ AND Yoshimi SAItō ${ }^{2}$ \\ 1 University of Heidelberg \\ Institute for Applied Mathematics \\ D-69120 Heidelberg \\ Germany \\ and \\ ${ }^{2}$ Department of Mathematics \\ University of Alabama at Birmingham \\ Birmingham, Alabama 35294 \\ U. S. A.
}

Typeset by $\mathcal{A M}_{\mathcal{M}}-\mathrm{T}_{\mathrm{E}} \mathrm{X}$ 


\section{$\S 1$. Introduction}

Let us consider the reduced wave equation

$$
-\Delta u(x)+q(x) u(x)=0
$$

on the domain $\Omega$ such that

$$
\Omega \supset E_{R_{0}}=\left\{x \in \mathbf{R}^{N}:|x|>R_{0}\right\},
$$

where $R_{0}>0$ and $N \geq 2$. Suppose that $q(x)$ has the form

$$
q(x)=-\ell(x)+s(x)
$$

where $\ell(x)$ a positive function, and $|s(x)|$ is supposed to be dominated by $\ell(x)$. The equation (1.1) has been studied extensively especially in the relation to the operator

$$
H_{1}=-\Delta+V(x)
$$

in $L_{2}(\Omega)$, or

$$
H_{2}=-\frac{1}{\mu(x)} \Delta
$$

in the weighted Hilbert space $L_{2}(\Omega ; \mu(x) d x)$ with boundary conditions on the boundary $\partial \Omega$ and at infinity. In this work we are concerned with the asymptotic behavior of the solution $u$ of the equation (1.1) at infinity. One of the important conclusions of the study is that we can establish the nonexistence of a class of (nontrivial) solutions of (1.1) which includes the $L_{2}$-solutions. And this result plays an important role in the attempt (the limiting absorption method, see, e.g., [1], [4]) to prove the existence

of the boundary value of the resolvent $\left(H_{1}-z\right)^{-1}$ or $\left(H_{2}-z\right)^{-1}$ of the operator $H_{1}$ or $H_{2}$ when the complex parameter $z$ approaches the real axis.

Consider the equation

$$
-\Delta u(x)+\left(-k^{2}+s(x)\right) u(x)=0,
$$

with $k>0$, i.e., $\ell(x)=k^{2}$ in (1.3). In the celebrated work Kato [9] he showed, among others, that, under the condition

$$
\tau \equiv(2 k)^{-1} \varlimsup_{|x| \rightarrow \infty}|x||s(x)|<1
$$


a nontrivial solution $u$ of the equation (1.1) satisfies

$$
\lim _{r \rightarrow \infty} r^{2 \tau+\epsilon} \int_{|x|=r}\left(|\nabla u(x)|^{2}+|u(x)|^{2}\right) d S=\infty
$$

for any $\epsilon>0$. One of the important features of the work [9] is that the coefficient $s(x)$ does not need to be spherically symmetric which makes the scope of application much wider than the preceding works (cf., e.g., Müller [10], Rellich [11]). Another important feature of [9] is that the method is based on differential inequalities satisfied by several functionals of the solution $u$ so that the problem was successfully treated as a local problem at infinity. As a result we do not need to use any boundary conditions at the boundary $\partial \Omega$ of $\Omega$ or at infinity such as radiation condition (cf., e.g., Wienholtz [15]). As is well-known this result has many applications. In Ikebe [3], in which the spectral theory and scattering theory for the Schrödinger operator $-\Delta+V(x)$ in $\mathbf{R}^{3}$ was developed under the conditin that

$$
V(x)=O\left(|x|^{-\gamma}\right) \quad(|x| \rightarrow \infty, \gamma>2),
$$

the result of Kato [9] was used to prove the existence of the boundary value of the Green function on the positive real axis as well as the nonexistence of the positive eigenvalues. After the work [9] various extensions and modifications were presented as many efforts were made to treat more general operators in a similar method. See e.g., Ikebe-Uchiyama [5] for Schrödinger operators with magnetic potentials, Jäger [6] for the second order elliptic operators, Weidmann [14] for the many body Schrödinger operators, and Ikebe-Saitō [4], Saitō[13] for Schrödinger operators with long-range potentials.

Now let us consider the case that $\ell(x)$ is a positive function which may be discontinuous. One of the motivations to consider such $\ell(x)$ comes from the study of the reduced wave equations in layered media. Consider the equation

$$
-\mu(x)^{-1} \Delta u-\lambda u=0 \quad\left(x \in \mathbf{R}^{N}\right)
$$

in layered media, where $\mu(x)$ is a positive function on $\mathbf{R}^{N}$. Suppose that the function $\mu(x)$ is a simple function with surfaces of discontinuity (separating surfaces) which may extend to infinity. Roach and Zhang [12] proved the nonexistence of the solution of the equation (1.10) under a geometric condition ("cone-like" discontinuity on the separating surface, see also [2]). Then Jäger and Saitō[7] proved a similar results under another geometric condition ("cylindrical" discontinuity) on the separating surfaces. In these works the method is not local at infinity, but some global integral identity of the solution $u$ are used. And the method seems to need some modifications in the case where $\mu$ is a perturbation such as

$$
\mu(x)=\mu_{0}(x)+\mu_{\ell}(x)+\mu_{s}(x),
$$


$\mu_{0}$ being a simple function, and $\mu_{\ell}(x)$ and $\mu_{s}(x)$ behaving like a long-range and short-range potentials at infinity, respectively (cf. [8]).

In this work we are going to obtain an extension of the result (1.8) by Kato [9] which can be applied the reduced wave equation (1.10) with $\mu(x)$ satisfying (1.11) as well as the equation (1.6) where $s(x)$ is the sum of a short-range potential and a long-range potential. Under the several assumptions (Assumptions 2.1, 4.2, 5.5 and 5.8 ) on the coefficient $q(x)$ the following (Theorem 5.10 in $\S 5$ ) will be proved:

Suppose that a solution $u$ of equation (1.1) satisfies

$$
\lim _{r \rightarrow \infty} \int_{|x|=r}\left\{\left|\frac{\partial u}{\partial r}\right|^{2}-\operatorname{Re}(q(x))|u|^{2}\right\} d S=0 .
$$

Then $u$ has a compact support.

Our method is a local method at infinity which is similar to the method of Kato [9]. As in [9], some type of differential inequalities on functionals of the solution $u$ will play important roles. However, we shall first establish the differential inequalities not in the ordinary sense but in the sense of distributions, and then they will be interpreted in the ordinary sense.

In $\S 2$ we define our reduced wave equation and give the main assumption (Assumption 2.1) on the coefficients. In $\S 3$ we introduce and evaluate the first functional $M^{+}(v, r)$. In order to complete the evaluation of $M^{+}(v, r)$, another functional $N(v, m, r)$ is introduced and evaluated in $\S 4$. $\S 5$ is devoted for proving the main theorem (Theorem 5.9). Some examples are discussed in $\S 6$. In $\S 7$ we shall discuss how our result can be applied to some reduced wave operators which were studied in [8]. A lemma on distributional derivative is given in Appendix.

Acknowledgement. This work was finished when the second author was visiting the University of Heidelberg for February 1997. Here he would like to thank Deutsche Forschungs Gemeinschaft for its support through SFB 359. Also the second author is thankful to Professor Willi Jäger for his kind hospitality during this period.

\section{$\S 2$. Schrödinger-type homogeneous equation}

Consider the homogeneous Schrödinger equation

$$
-\Delta u(x)+q(x) u(x)=0 \quad\left(x \in E_{R_{0}}\right),
$$


where $R_{0}>0$, and

$$
E_{R}=\left\{x \in \mathbf{R}^{N}:|x|>R\right\} .
$$

Let $S^{N-1}$ be the unit sphere of $\mathbf{R}^{N}$. We set $X=L_{2}\left(S^{N-1}\right)$ and the inner product and norm of $X$ is denoted by $($,$) and ||$, respectively.

Assumption 2.1. (i) Let $N$ be an integer such that $N \geq 2$. Let $u \in H^{2}\left(E_{R_{0}}\right)_{\text {loc }}$, $R_{0}>0$, be a solution of the equation (2.1), where $q(x)$ is a complex-valued, measurable, locally bounded function on $E_{R_{0}}$.

(ii) Set

$$
Q(x)=q(x)+\frac{(N-1)(N-3)}{4 r^{2}} .
$$

(ii-a) Then $Q(x)$ is decomposed as

$$
Q(x)=Q_{0}(x)+Q_{1}(x)
$$

where $Q_{0}(x)$ is a real-valued, measurable, locally bounded function on $E_{R_{0}}$ such that

$$
Q_{0}(x) \leq 0
$$

and $Q_{1}(x)$ is a complex-valued, measurable, locally bounded function on $E_{R_{0}}$.

(ii-b) For any $x \in X=L_{2}\left(S^{N-1}\right),\left(Q_{0}(r \cdot) x, x\right)$ has the right limit for all $r>R_{0}$ as a function of $r=|x|$.

(ii-c) There exist $h_{0}>0$ and, for $0<h<h_{0}$, a real-valued, measurable function $Q_{0 r}(x ; h)$ on $E_{R_{0}}$ such that

$$
\sup \left\{\left|Q_{0 r}(x ; h)\right| / x \in G, 0<h<h_{0}\right\}<\infty
$$

for any compact set $G \subset E_{R_{0}}$,

$$
\begin{aligned}
\frac{1}{h}\left(\left\{Q_{0}((r+h) \cdot)-Q_{0}(r \cdot)\right\} \phi, \phi\right) & \leq\left(Q_{0 r}(r \cdot ; h) \phi, \phi\right) \\
& \left(\phi \in X, r>R_{0}, 0<h \leq h_{0}\right),
\end{aligned}
$$

and the limit

$$
\lim _{h \downarrow 0}\left(Q_{0 r}(r \cdot ; h) \phi, \phi\right)=\left(Q_{0 r}(r \cdot) \phi, \phi\right) \quad(\phi \in X)
$$

exists with a real-valued, measurable, locally bounded function $Q_{0 r}(x)$ on $E_{R_{0}}$. 
(iii) There exists a positive, measurable function $h(r)$ defined on $\left(R_{0}, \infty\right)$ such that

(iii-a)

$$
h(r) \leq \frac{2}{r} \quad\left(r>R_{0}\right)
$$

(iii-b) and, setting

$$
\left\{\begin{array}{l}
a(r)=h^{-1}(r) \sup _{|x|=r}\left|Q_{1}(x)\right| \\
b(r)=\inf _{|x|=r}\left[-\left(Q_{0}(x)+h^{-1}(r) Q_{0 r}(x)\right)\right]
\end{array}\right.
$$

where $h^{-1}(r)=1 / h(r)$, we have

$$
a(r)^{2} \leq b(r) \quad\left(r>R_{0}\right)
$$

In order to transform the equation (2.1) into a differential equation on $\left(R_{0}, \infty\right)$ with operator-valued coefficients, we give the following

Definition 2.2. (i) For $r>R_{0}$ define a selfadjoint operator $B(r)$ in $X$ by

$$
\left\{\begin{array}{c}
D(B(r))=D\left(\Lambda_{N}\right), \\
B(r)=-r^{-2} \Lambda_{N},
\end{array}\right.
$$

where $D(T)$ is the domain of $T$, and $\Lambda_{N}$ is the (selfadjoint realization of) LaplaceBeltrami operator on $S^{N-1}$.

(ii) For $r>R_{0}$ define a bounded operators $C_{0}(r), C_{0 r}(r ; h), C_{0 r}(r)$ and $C_{1}(r)$ on $X$ by

$$
\left\{\begin{array}{l}
C_{0}(r)=Q_{0}(r \cdot) \times, \\
C_{0 r}(r ; h)=Q_{0 r}(r \cdot ; h) \times, \\
C_{0 r}(r)=Q_{0 r}(r \cdot) \times \\
C_{1}(r)=Q_{1}(r \cdot) \times .
\end{array}\right.
$$

Proposition 2.3. Let $u$ be a solution of the equation (2.1) and let $v$ be as in Assumption 2.1, (ii). Let $J=\left(R_{0}, \infty\right)$. Then,

(i) $v \in C^{1}(J, X)$.

(ii) $v(r) \in D\left(\left(-\Lambda_{N}\right)^{1 / 2}\right)$ for $r \in J$. 
(iii) We have

$$
\int_{r}^{s}\left\{\left|v^{\prime}(r)\right|^{2}+\left|B^{1 / 2}(r) v(r)\right|^{2}\right\} d r<\infty \quad\left(R_{0}<r<s<\infty\right),
$$

where $v^{\prime}(r)=d v(r) / d r$ and $B^{1 / 2}(r)=B(r)^{1 / 2}$.

(iv) $v(r) \in D\left(\Lambda_{N}\right)$ for almost all $r \in J$, and $B v \in L_{2}((r, s), X)$ for $R_{0}<r<$ $s<\infty$.

(v) $v^{\prime}(r) \in C_{\mathrm{ac}}([r, s], X)$ for $R_{0}<r<s<\infty$, where $C_{\mathrm{ac}}([r, s], X)$ is all $X$-valued absolutely continuous functions on $[r, s]$. There exists the weak derivative $v^{\prime \prime}(r)$ of $v^{\prime}(r)$ for $r \in J$.

(vi) $v^{\prime}(r) \in D\left(\left(-\Lambda_{N}\right)^{1 / 2}\right)$ for almost all $r \in J$, and $B^{1 / 2} v^{\prime} \in L_{2}((r, s), X)$ for $R_{0}<r<s<\infty$.

(vii) $B^{1 / 2} v \in C_{\mathrm{ac}}([r, s], X)$ for $R_{0}<r<s<\infty$, and we have

$$
\begin{aligned}
\frac{d}{d r}\left(B^{1 / 2}(r) v(r), B^{1 / 2}(r) v(r)\right)=- & \frac{2}{r}\left(B^{1 / 2}(r) v(r), B^{1 / 2}(r) v(r)\right) \\
& +2 \operatorname{Re}\left(B^{1 / 2}(r) v^{\prime}(r), B^{1 / 2}(r) v(r)\right)
\end{aligned}
$$

for almost all $r \in J$.

(viii) We have

$$
-v^{\prime \prime}(r)+B(r) v(r)+C_{0}(r) v(r)+C_{1}(r) v(r)=0
$$

in $X$ for almost all $r \in J$.

Proof. See [13], Proposition 1.3.

Proposition 2.4. Suppose that $Q_{0 r}(x)$ satisfies Assumption 2.1, (ii-b) and (ii-c). Let $\eta \in C^{1}(J, X)$. Let

$$
g(r)=\frac{d}{d r}\left(C_{0}(r) \eta(r), \eta(r)\right)
$$

be the derivative of $f(r)=\left(C_{0}(r) \eta(r), \eta(r)\right)$ in the sense of distributions on $\left(R_{0}, \infty\right)$. Then we have

$$
g(r) \leq\left(C_{0 r}(r) \eta(r), \eta(r)\right)+2 \operatorname{Re}\left(C_{0}(r) \eta(r), \eta^{\prime}(r)\right)
$$

where the inequality (2.18) should be taken in the sense of distributions on $\left(R_{0}, \infty\right)$ again. 
Proof. (I) Let $\varphi$ be a nonnegative $C_{0}^{\infty}\left(\left(R_{0}, \infty\right)\right)$ function. Then, by definition

$$
\begin{aligned}
<g, \varphi> & =-<f, \varphi^{\prime}> \\
& =-\lim _{h \uparrow 0} \int_{R_{0}}^{\infty} f(r) \frac{\varphi(r+h)-\varphi(r)}{h} d r \\
& =\lim _{h \downarrow 0} \frac{1}{h} \int_{R_{0}}^{\infty}(f(r+h)-f(r)) \varphi(r) d r
\end{aligned}
$$

where $<$, $>$ denotes the dual pair bracketing.

(II) Here we have

$$
\begin{array}{r}
f(r+h)-f(r)=\left(C_{0}(r+h) \eta(r+h), \eta(r+h)\right)-\left(C_{0}(r) \eta(r), \eta(r)\right) \\
=\left(\left\{C_{0}(r+h)-C_{0}(r)\right\} \eta(r+h), \eta(r+h)\right) \\
+\left(C_{0}(r) \eta(r+h), \eta(r+h)-\eta(r)\right) \\
+\left(\eta(r+h)-\eta(r), C_{0}(r) \eta(r)\right)
\end{array}
$$

and hence, using (2.7) in (ii-b) of Assumption 2.1, we obtain

$$
\begin{array}{r}
\frac{1}{h}(f(r+h)-f(r)) \leq\left(C_{0 r}(r ; h) \eta(r+h), \eta(r+h)\right) \\
+\left(C_{0}(r) \eta(r+h), \frac{1}{h}(\eta(r+h)-\eta(r))\right) \\
+\left(\frac{1}{h}(\eta(r+h)-\eta(r)), C_{0}(r) \eta(r)\right) .
\end{array}
$$

(III) It is easy to see from (2.8) in (ii-c) of Assumption 2.1 and (i) of Proposition 2.3 that the right-hand side of $(2.21)$ converges to

$$
g_{0}(r) \equiv\left(C_{0 r}(r) \eta(r), \eta(r)\right)+2 \operatorname{Re}\left(C_{0}(r) \eta(r), \eta(r)\right)
$$

boundedly on any compact interval in $\left(R_{0}, \infty\right)$ as $h \downarrow 0$. Therefore, noting that $\varphi \geq 0$, we have

$$
<g, \varphi>\leq \int_{R_{0}}^{\infty} g_{0}(r) \varphi(r) d r=<g_{0}, \varphi>,
$$

which completes the proof. 


\section{$\S 3$. The evaluation of the functional $M^{+}(v, r)$}

Let $v=v(r \cdot)$ be as in (2.5). Then we are going to define the functional $M^{+}(v, r)$ by

Definition 3.1. Let $v$ be as in (ii-b) of Assumption 2.1. Then set

$$
M^{+}(v, r)=\left|v^{\prime}(r)\right|^{2}-\left(C_{0}(r) v(r), v(r)\right)-\left|B^{1 / 2}(r) v(r)\right|^{2}
$$

for $r>R_{0}$.

Proposition 3.2. Suppose that Assumption 2.1 is satisfied. Let $M^{+}(v, r)$ be as in Definition 3.1.

(i) Then $M^{+}(v, r)$ is a real-valued, locally bounded function on $J=\left(R_{0}, \infty\right)$. Further $M^{+}(v, r)$ is right continuous with its left limit for $r \in J$.

(ii) We have

$$
\frac{d}{d r} M^{+}(v, r) \geq-h(r) M^{+}(v, r) \quad\left(r>R_{0}\right),
$$

where the inequality (3.2) should be taken in the sense of distributions on $\left(R_{0}, \infty\right)$.

Proof. (i) follows from Assumption 2.1, (ii) and Proposition 2.3. From Propositions 2.3 and 2.4 we see that

$$
\begin{aligned}
\frac{d}{d r} M^{+}(v, r) \geq 2 \operatorname{Re} & \left(v^{\prime \prime}(r), v^{\prime}(r)\right) \\
& -\left(C_{0 r}(r) v(r), v(r)\right)-2 \operatorname{Re}\left(C_{0}(r) v(r), v^{\prime}(r)\right) \\
+ & \frac{2}{r}(B(r) v(r), v(r))-2 \operatorname{Re}\left(B(r) v(r), v^{\prime}(r)\right)
\end{aligned}
$$

in the sense of distributions on $\left(R_{0}, \infty\right)$. Using $(2.16)$, we have from (3.3)

$$
\begin{aligned}
& \frac{d}{d r} M^{+}(v, r) \geq-\left(C_{0 r}(r) v(r), v(r)\right)+2 \operatorname{Re}\left(C_{1}(r)\right.\left.v(r), v^{\prime}(r)\right) \\
&+ \frac{2}{r}(B(r) v(r), v(r)) \\
&=-h(r) M^{+}(v, r) \quad \\
&+h(r)\left[\left|v^{\prime}(r)\right|^{2}-\left(C_{0}(r) v(r), v(r)\right)-(B(r) v(r), v(r))\right] \\
&-\left(C_{0 r}(r) v(r), v(r)\right)+ 2 \operatorname{Re}\left(C_{1}(r) v(r), v^{\prime}(r)\right) \\
&+ \frac{2}{r}(B(r) v(r), v(r))
\end{aligned}
$$


Thus, using $a(r)$ and $b(r)$ defined by (2.10), and taking note of (2.9) in Assumption 2.1 , we have

$$
\begin{aligned}
\frac{d}{d r} M^{+}(v, r) \geq-h(r) & M^{+}(v, r) \\
& +h(r)\left[\left|v^{\prime}(r)\right|^{2}-2 a(r)\left|v^{\prime}(r)\right||v(r)|+b(r)|v(r)|^{2}\right] .
\end{aligned}
$$

It follows from (2.11) in Assumption 2.1, that is, $a(r)^{2} \leq b(r)$, that (3.5) implies (3.2), which completes the proof.

Proposition 3.3. Suppose that Assumption 2.1 is satisfied. For $R_{1}>R_{0}$ we have

$$
M^{+}(v, r) \geq \exp \left(-\int_{R_{1}}^{r} h(t) d t\right) M^{+}\left(v, R_{1}\right) \quad\left(r \geq R_{1}\right) .
$$

Proof. It follows from Proposition 3.2 that

$$
\begin{aligned}
\exp & \left(\int_{R_{1}}^{r} h(t) d t\right) \frac{d}{d r} M^{+}(v, r) \\
& +h(r) \exp \left(\int_{R_{1}}^{r} h(t) d t\right) M^{+}(v, r) \geq 0 \quad\left(r>R_{1}\right),
\end{aligned}
$$

and hence

$$
\frac{d}{d r}\left[\exp \left(\int_{R_{1}}^{r} h(t) d t\right) M^{+}(v, r)\right] \geq 0 \quad\left(r>R_{1}\right)
$$

in the sense of distributions on $\left(R_{1}, \infty\right)$. The inequality (3.6) follows from (3.8) and Lemma A of Appendix.

\section{$\S 4$. The evaluation of the functional $N(v, m, r)$}

Using $M^{+}(v, r)$, we are going to define another functional which will be used to evaluate $M^{+}(v, r)$ in $\S 5$. 
Definition 4.1. (i) Set

$$
N(v, m, r)=M^{+}(w, r)+(m(m+1)-F(r)) r^{-2}|w|^{2} \quad\left(w=r^{m} v\right),
$$

where $m$ is a positive number and $F(r)$ is a positive $C^{1}$ function on $\left(R_{0}, \infty\right)$.

(ii) For $r>R_{0}$ define a bounded operators $C_{R}(r)$ on $X$ by

$$
C_{R}(r)=\operatorname{Re}(Q(r \cdot)) \times=\left(Q_{0}(r \cdot)+\operatorname{Re}\left(Q_{1}(r \cdot)\right) \times .\right.
$$

Set

$$
M(v, r)=\left|v^{\prime}(r)\right|^{2}-\left(C_{R}(r) v(r), v(r)\right) .
$$

(iii) For $r>R_{0}$ we set

$$
p(r)=\inf _{|x|=r}\left[-\left(2 Q_{0}(x)+r Q_{0 r}(x)\right)\right] .
$$

Assumption 4.2. The function $F(r)$ introduced in Definition 4.1 satisfies the following (i) (iii):

(i) There exists a positive constant $c_{0}$ such that

$$
F^{2}(r) \leq c_{0} r^{4} h^{2}(r) b(r) \quad\left(r>R_{0}\right),
$$

where $b(r)$ is given in (2.10), $F^{2}(r)=F(r)^{2}$, and $h^{2}(r)=h(r)^{2}$.

(ii) We have $F(r) \rightarrow \infty$ as $r \rightarrow \infty$.

(iii) There exists a positive constant $c_{1}$ such that

$$
F_{r}(r) \equiv \frac{d}{d r} F(r) \leq c_{1} r^{-1} \quad\left(r>R_{0}\right) .
$$

Proposition 4.3. (i) Let $b(r)$ be given by (2.10) and assume that $h(r)$ satisfies the inequality (2.9) and that $Q_{0}(x)$ is nonpositive. Then,

$$
r^{2} h^{2}(r) b(r) \leq 2 p(r) \quad\left(r>R_{0}\right) .
$$

(ii) Assume that the inequality (2.11) holds. Then,

$$
\left(r \sup _{|x|=r}\left|Q_{1}(x)\right|\right)^{2} \leq 2 p(r) \quad\left(r>R_{0}\right) .
$$

(iii) Suppose that the inequality $(4,5)$ holds. Then,

$$
r^{-2} F^{2}(r) \leq 2 c_{0} p(r) \quad\left(r>R_{0}\right) .
$$


Proof. Since $0<r h(r) \leq 2$ and $-Q_{0}(x) \geq 0$, we have

$$
\begin{aligned}
r^{2} h^{2}(r)[ & \left.-\left(Q_{0}(x)+h^{-1}(r) Q_{0 r}(x)\right)\right] \\
& =r h(r)\left[r h(r)\left(-Q_{0}(x)\right)+r\left(-Q_{0 r}(x)\right)\right] \\
& \leq 2\left[2\left(-Q_{0}(x)\right)+r\left(-Q_{0 r}(x)\right)\right] \\
& =2\left[-\left(2 Q_{0}(x)+r Q_{0 r}(x)\right)\right]
\end{aligned}
$$

which implies (4.7). It follows from (2.11) and (4.7) that

$$
\begin{aligned}
& \left(r \sup _{|x|=r}\left|Q_{1}(x)\right|\right)^{2} \\
& \quad=r^{2} h^{2}(r)\left(h^{-1}(r) \sup _{|x|=r}\left|Q_{1}(x)\right|\right)^{2} \\
& \quad \leq r^{2} h^{2}(r) b(r) \\
& \quad \leq 2 p(r) .
\end{aligned}
$$

From (4.5) and (4.7) we obtain

$$
F^{2}(r) \leq c_{0} r^{4} h^{2}(r) b(r) \leq c_{0} r^{2}(2 p(r))=r^{2}\left(2 c_{0} p(r)\right)
$$

for $r>R_{0}$, which implies (4.9).

Proposition 4.4. Suppose that Assumptions 2.1 and 4.2 hold. Then there exist $m_{0}>0$ and $r_{0}>R_{0}$ such that

$$
\frac{d}{d r}\left(r^{2} N(v, m, r)\right) \geq 0 \quad\left(m \geq m_{0}\right)
$$

in the sense of distributions on $\left(r_{0}, \infty\right)$.

Proof. (I) By definition $w=r^{m} v$ satisfies

$$
\left\{\begin{aligned}
w^{\prime} & =r^{m} v^{\prime}+m r^{m-1} v=r^{m} v^{\prime}+m r^{-1} w \\
w^{\prime \prime} & =r^{m} v^{\prime \prime}+2 m r^{m-1} v^{\prime}+m(m-1) r^{m-2} v \\
& =r^{m} v^{\prime \prime}+2 m r^{-1}\left(w^{\prime}-m r^{-1} w\right)+m(m-1) r^{-2} w \\
& =r^{m} v^{\prime \prime}+2 m r^{-1} w^{\prime}-m(m+1) r^{-2} w \\
& =r^{m}\left(B v+C_{0} v+C_{1} v\right)+2 m r^{-1} w^{\prime}-m(m+1) r^{-2} w
\end{aligned}\right.
$$


and hence we have

$$
-w^{\prime \prime}+2 m r^{-1} w^{\prime}+\left(B+C_{0}+C_{1}-m(m+1) r^{-2}\right) w=0 .
$$

(II) Set

$$
g(r, m)=(m(m+1)-F(r)) r^{-2} .
$$

Then, using (4.15) and Proposition 2.4, we have

$$
\begin{gathered}
r^{-2} \frac{d}{d r}\left(r^{2} N(v, m, r)\right) \\
\geq 2 r^{-1}\left(\left|w^{\prime}\right|^{2}-\left(C_{0} w, w\right)-(B w, w)+(g w, w)\right) \\
\quad+2 \operatorname{Re}\left(w^{\prime \prime}-C_{0} w-B w+g w, w^{\prime}\right)+\frac{2}{r}\left|B^{1 / 2} w\right|^{2} \\
-\left(C_{0 r} w, w\right)+\left(g_{r} w, w\right) \\
=2 r^{-1}\left(\left|w^{\prime}\right|^{2}-\left(C_{0} w, w\right)-(B w, w)+(g w, w)\right) \\
+2 \operatorname{Re}\left(2 m r^{-1} w^{\prime}+C_{1} w-m(m+1) r^{-2} w+g w, w^{\prime}\right) \\
\quad+\frac{2}{r}\left|B^{1 / 2} w\right|^{2}-\left(C_{0 r} w, w\right)+\left(g_{r} w, w\right) \\
=2(1+2 m) r^{-1}\left|w^{\prime}\right|^{2}+\left(2 r^{-1} g+g_{r}\right)|w|^{2} \\
+r^{-1}\left(\left[-2 C_{0}-r C_{0 r}\right] w, w\right) \\
+2 \operatorname{Re}\left(C_{1} w-m(m+1) r^{-2} w+g w, w^{\prime}\right),
\end{gathered}
$$

where $g_{r}=d g / d r$. Note that

$$
\left\{\begin{array}{l}
2 r^{-1} g(r, m)+g_{r}(r, m)=-F_{r}(r) r^{-2} \\
g(r, m)-m(m+1) r^{-2}=-F(r) r^{-2}
\end{array}\right.
$$

Then the above inequality (4.17) can be rewritten as

$$
\begin{aligned}
r^{-2} \frac{d}{d r} & \left(r^{2} N(v, m, r)\right) \\
\geq 2(1+2 m) r^{-1}\left|w^{\prime}\right|^{2} & +r^{-1}\left(p(r)-r^{-1} F_{r}(r)\right)|w|^{2} \\
& -2 \operatorname{Re}\left(\left(r^{-2} F(r)-C_{1}\right) w, w^{\prime}\right)
\end{aligned}
$$

where $p(r)$ is as in (4.4). 
(III) It follows from Assumption 4.2, (iii) and Proposition 4.3, (ii), (iii) that

$$
\left\{\begin{aligned}
p(r)-r^{-1} F_{r}(r) & \geq p(r)-c_{1} r^{-2}=r^{-2}\left(r^{2} p(r)-c_{1}\right), \\
\left|r^{-2} F(r)-Q_{1}(x)\right| & \leq r^{-1}\left(2 c_{0} p(r)\right)^{1 / 2}+r^{-1}(2 p(r))^{1 / 2} \\
& =c_{2} r^{-1} p^{1 / 2}(r) \quad\left(c_{2}=\sqrt{2 c_{0}}+\sqrt{2}\right) .
\end{aligned}\right.
$$

Further, from (iii) of Proposition 4.3 and (ii) of Assumption 4.2 we see that

$$
r^{2} p(r) \geq\left(2 c_{0}\right)^{-1} F^{2}(r) \rightarrow \infty \quad(r \rightarrow \infty),
$$

and hence there exists $r_{0}>R_{0}$ such that

$$
r^{-2}\left(r^{2} p(r)-c_{1}\right)=p(r)\left(1-\frac{c_{1}}{r^{2} p(r)}\right) \geq 2^{-1} p(r)
$$

for $r \geq r_{0}$. Thus, we obtain from (5.19)

$$
\begin{aligned}
r^{-2} \frac{d}{d r} & \left(r^{2} N(v, m, r)\right) \\
\geq 2(1+2 m) r^{-1}\left|w^{\prime}\right|^{2} & +2^{-1} r^{-1} p(r)|w|^{2} \\
& -2 c_{2} r^{-1} p^{1 / 2}(r)\left|w^{\prime}\right||w| \\
=r^{-1}\left[2(1+2 m)\left|w^{\prime}\right|^{2}\right. & +2^{-1} p(r)|w|^{2} \\
& \left.-2 c_{2} p^{1 / 2}(r)\left|w^{\prime}\right||w|\right]
\end{aligned}
$$

for $r \geq r_{0}$. Therefore, there exists a sufficiently large $m_{0}>0$ such that

$$
r^{2} \frac{d}{d r}\left(r^{2} N(v, m, r)\right) \geq 0
$$

for $r \geq r_{0}$ and $m \geq m_{0}$, which completes the proof. 


\section{$\S 5$. Uniqueness Theorem}

We are going to prove our main theorem (Theorem 5.10) which shows, under Assumptions 2.1 and 4.1, and some additional conditions (Assumptions 5.5 and 5.8), that the solution $u$ has compact support if $u$ satisfies

$$
\varliminf_{r \rightarrow \infty} \int_{|x|=r}\left\{\left|\frac{\partial u}{\partial r}\right|^{2}-\operatorname{Re}(q(x))|u|^{2}\right\} d S=0 .
$$

Proposition 5.1. Suppose that Assumptions 2.1 and 4.2 hold. Suppose that the support of $u$ is unbounded. Let $r_{0}$ and $m_{0}$ be as in Proposition 4.4. Then there exist $m_{1} \geq m_{0}$ and $r_{1} \geq r_{0}$ such that

$$
N\left(v, m_{1}, r\right)>0 \quad\left(r \geq r_{1}\right) .
$$

Proof. Since the support of $u$ is assumed to be unbounded, there exists $r_{1} \geq r_{0}$ such that $\left|v\left(r_{1}\right)\right|>0$. Since

$$
\begin{aligned}
& r_{1}^{-2 m} N\left(v, m, r_{1}\right) \\
& =r_{1}^{-2 m}\left\{\left|w^{\prime}\left(r_{1}\right)\right|^{2}-\left(C_{0}\left(r_{1}\right) w\left(r_{1}\right), w\left(r_{1}\right)\right)\right. \\
& \left.\quad-\left|B^{1 / 2}\left(r_{1}\right) w\left(r_{1}\right)\right|^{2}+\left(m(m+1)-F\left(r_{1}\right)\right)\left|w\left(r_{1}\right)\right|^{2}\right\} \\
& \geq-\left(C_{0}\left(r_{1}\right) v\left(r_{1}\right), v\left(r_{1}\right)\right) \\
& \quad-\left|B^{1 / 2}\left(r_{1}\right) v\left(r_{1}\right)\right|^{2}+\left(m(m+1)-F\left(r_{1}\right)\right)\left|v\left(r_{1}\right)\right|^{2},
\end{aligned}
$$

we can choose a sufficiently large $m_{1} \geq m_{0}$ so that

$$
r_{1}^{-2 m_{1}} N\left(v, m_{1}, r_{1}\right)>0 \text {, or } r_{1}^{2} N\left(v, m_{1}, r_{1}\right)>0 \text {. }
$$

Note that, by (ii)-2 of Assumption 2.1, $N(r, m, v)$ is right-continuous. Then the inequality (5.4) is combined with (4.13) and Lemma A in Appendix to see that $r^{2} N\left(r, m_{1}, v\right)>0$ on $\left[r_{1}, \infty\right)$, which completes the proof.

Definition 5.2. Suppose that Assumptions 2.1 and 4.2 hold. Suppose that the support of $u$ is unbounded. Let $F(r)$ and $m_{1}$ be given in Definition 4.1 and Proposition 5.1, respectively. Then we introduce the following two alternative cases:

Case I: There exists an infinite sequence $\left\{r_{\ell}^{\prime}\right\}$ such that $R_{0}<r_{\ell}^{\prime}, r_{\ell}^{\prime} \rightarrow \infty$ as $\ell \rightarrow \infty$, and

$$
2 \operatorname{Re}\left(v^{\prime}\left(r_{\ell}^{\prime}\right), v\left(r_{\ell}^{\prime}\right)\right) \leq\left(2 m_{1} r_{\ell}^{\prime}\right)^{-1} F\left(r_{\ell}^{\prime}\right)\left|v\left(r_{\ell}^{\prime}\right)\right|^{2}
$$

for all $\ell=1,2, \cdots$.

Case II: There exists $r_{2}>r_{1}$ such that

$$
2 \operatorname{Re}\left(v^{\prime}(r), v(r)\right)>\left(2 m_{1} r\right)^{-1} F(r)|v(r)|^{2} . \quad\left(r \geq r_{2}\right),
$$

where $r_{1}$ is as in Proposition 5.1. 
Proposition 5.3. Suppose that Assumptions 2.1 and 4.2 hold. Suppose that the support of $u$ is unbounded. Suppose that Case I in Definition 5.2 holds. Then there exists an infinite sequence $\left\{r_{\ell}^{\prime \prime}\right\}$ such that $R_{0}<r_{\ell}^{\prime \prime}, r_{\ell}^{\prime \prime} \rightarrow \infty$ as $\ell \rightarrow \infty$, and

$$
M^{+}\left(v, r_{\ell}^{\prime \prime}\right)>0 \quad(\ell=1,2, \cdots) .
$$

Proof. Let $\left\{r_{\ell}^{\prime}\right\}$ be as in Case I of Definition 5.2. Let $w=r^{m_{1}} v$, where $m_{1}$ is as in Proposition 5.1. Then we have for $r=r_{\ell}^{\prime}$

$$
\begin{aligned}
r^{-2 m_{1}}\left|w^{\prime}\right|^{2} & =\left|v^{\prime}+m_{1} r^{-1} v\right|^{2} \\
& =\left|v^{\prime}\right|^{2}+2 m_{1} r^{-1} \operatorname{Re}\left(v^{\prime}, v\right)+m_{1}^{2} r^{-2}|v|^{2} \\
& \leq\left|v^{\prime}\right|^{2}+m_{1} r^{-1}\left(2 m_{1} r\right)^{-1} F(r)|v|^{2}+m_{1}^{2} r^{-2}|v|^{2} \\
& =\left|v^{\prime}\right|^{2}+\left(2^{-1} F(r)+m_{1}^{2}\right) r^{-2}|v|^{2} .
\end{aligned}
$$

Let $r_{1}$ be as in Proposition 5.1. For $r=r_{\ell}^{\prime}$ such that $r_{\ell}^{\prime} \geq r_{1}$, it follows that

$$
\begin{aligned}
0<N\left(v, m_{1}, r\right) & =M^{+}(w, r)+\left(m_{1}\left(m_{1}+1\right)-F(r)\right) r^{-2}|w|^{2} \\
\leq r^{2 m_{1}}\left\{\left|v^{\prime}\right|^{2}\right. & \left.+\left(2^{-1} F(r)+m_{1}^{2}\right) r^{-2}|v|^{2}\right\} \\
& -r^{2 m_{1}}\left\{\left(C_{0} v, v\right)+(B v, v)\right\} \\
& +r^{2 m_{1}}\left(m_{1}\left(m_{1}+1\right)-F(r)\right) r^{-2}|v|^{2} \\
= & r^{2 m_{1}}\left\{M^{+}(v, r)+\left(m_{1}\left(2 m_{1}+1\right)-2^{-1} F(r)\right) r^{-2}|v|^{2}\right\} .
\end{aligned}
$$

Since $F(r) \rightarrow \infty$ as $r \rightarrow \infty$, there exists a positive integer $\ell_{0}$ such that

$$
\left.m_{1}\left(2 m_{1}+1\right)-2^{-1} F\left(r_{\ell}^{\prime}\right)\right)<0 \quad\left(\ell \geq \ell_{0}\right) .
$$

Therefore we have only to define $r_{\ell}^{\prime \prime}$ by

$$
r_{\ell}^{\prime \prime}=r_{\ell_{0}+\ell}^{\prime} \quad(\ell=1,2, \cdots),
$$

which completes the proof.

Proposition 5.4. Suppose that Assumptions 2.1 and 4.2 hold. Suppose that the support of $u$ is unbounded. Suppose that Case II in Definition 5.2 holds. Suppose, in addition, that

$$
\operatorname{Re} Q(x) \leq 0 \quad\left(x \in E_{R_{0}}\right) .
$$

Then there exist $r_{3}>R_{0}$ and a positive constant $c_{2}$ such that

$$
M(v, r) \geq c_{2} \quad\left(r \geq r_{3}\right),
$$

where $M(v, r)$ is given by (4.3). 
Proof. Since $F(r) \rightarrow \infty$ as $r \rightarrow \infty$, there exists $r_{4}>R_{0}$ such that

$$
\frac{F(r)}{2 m_{1}} \geq 2 \quad\left(r \geq r_{4}\right) .
$$

Then it follows from (5.6) that

$$
\frac{d}{d r}|v(r)|^{2} \geq 2 r^{-1}|v(r)|^{2} \quad\left(r \geq r_{4}\right) .
$$

Let $r_{3}$ be such that $r_{3} \geq r_{4}$ and $\left|v\left(r_{3}\right)\right|>0$. Then, since

$$
\frac{d}{d r}\left(r^{-2}|v(r)|^{2}\right)=r^{-2}\left(\frac{d}{d r}|v(r)|^{2}-2 r^{-1}|v(r)|^{2}\right) \geq 0 \quad\left(r \geq r_{4}\right),
$$

we have

$$
r^{-2}|v(r)|^{2} \geq r_{3}^{-2}\left|v\left(r_{3}\right)\right|^{2}>0 \quad\left(r \geq r_{3}\right) .
$$

Also, using (5.6) and (5.14) again, we see that

$$
2 r^{-1}|v(r)|^{2} \leq\left(2 m_{1} r\right)^{-1} F(r)|v(r)|^{2} \leq 2|v(r)|\left|v^{\prime}(r)\right|,
$$

or

$$
r^{-1}|v(r)| \leq\left|v^{\prime}(r)\right|
$$

for $r \geq r_{4}$. Thus, it follows from (5.17) and (5.19) that

$$
\left|v^{\prime}(r)\right|^{2} \geq r^{-2}|v(r)|^{2} \geq r_{2}^{-2}\left|v\left(r_{3}\right)\right|^{2}>0
$$

for $r \geq r_{3}$, which is combined with (5.12) to obtain (5.13).

Assumption 5.5. (i) Let $h(r)$ be as above. Then $h \in L_{1}\left(\left(R_{0}, \infty\right)\right)$.

(ii) There exists a constant $\beta \in(0,1)$ such that

$$
0 \geq \beta Q_{0}(x) \geq \operatorname{Re}(Q(x)) \quad\left(x \in E_{R_{0}}\right) .
$$

Theorem 5.6. Suppose that Assumptions 2.1, 4.2 and 5.5 hold. Suppose that the support of $u$ is unbounded. Then there exist a positive constant $c_{3}$ and $R_{2}>R_{0}$ such that

$$
M(v, r) \geq c_{3} \quad\left(r \geq R_{2}\right) .
$$


Proof. Note that all the assumptions that are necessary for the conclusions of Propositions 3.2, 3.3, 4.3, 4.4, 5.15 .3 and 5.4 are satisfied. Suppose that Case I of Definition 5.2 is satisfied. Then, by Proposition 5.3 there exists $R_{2}^{\prime}>R_{0}$ such that $M^{+}\left(v, R_{2}^{\prime}\right)>0$. Therefore, setting $R_{1}=R_{2}^{\prime}$ in Proposition 3.3, we have for $r \geq R_{2}^{\prime}$,

$$
\begin{aligned}
M^{+}(v, r) & \geq \exp \left(-\int_{R_{3}^{\prime}}^{r} h(t) d t\right) M^{+}\left(v, R_{2}^{\prime}\right) \\
& \geq \exp \left(-\int_{R_{3}^{\prime}}^{\infty} h(t) d t\right) M^{+}\left(v, R_{2}^{\prime}\right) .
\end{aligned}
$$

Since we have from (5.21)

$$
\begin{aligned}
M(v, r) & =\left|v^{\prime}(r)\right|^{2}-\left(C_{R}(r) v(r), v(r)\right) \\
& \geq\left|v^{\prime}(r)\right|^{2}-\beta\left(C_{0}(r) v(r), v(r)\right) \\
& \geq \beta\left|v^{\prime}(r)\right|^{2}-\beta\left(C_{0}(r) v(r), v(r)\right)-\beta\left|B^{1 / 2}(r) v(r)\right|^{2} \\
& =\beta M^{+}(v, r),
\end{aligned}
$$

it follows from (5.23) that

$$
M(v, r) \geq c_{3}^{\prime} \quad\left(r \geq R_{2}^{\prime}\right)
$$

with

$$
c_{3}^{\prime}=\beta \exp \left(-\int_{R_{3}^{\prime}}^{\infty} h(t) d t\right) M^{+}\left(v, R_{2}^{\prime}\right) .
$$

Suppose that Case II of Definition 5.2 is satisfied. Then from Proposition 5.4 we have

$$
M(v, r) \geq c_{2} \quad\left(r \geq r_{3}\right),
$$

where $c_{2}$ and $r_{3}$ are as in Proposition 5.4. Now set

$$
\left\{\begin{array}{l}
c_{3}=\min \left\{c_{3}^{\prime}, c_{2}\right\}, \\
R_{2}=\max \left\{R_{2}^{\prime}, r_{3}\right\} .
\end{array}\right.
$$

Then (5.22) follows, which completes the proof.

Corollary 5.7. Suppose that Assumptions 2.1, 4.2 and 5.8 hold. Suppose that

$$
\varliminf_{r \rightarrow \infty} M(v, r)=0 .
$$

Then u has compact support. 
In order to show our main theorem (Theorem 5.10) we need one more assumption.

Assumption 5.8. We have

$$
\lim _{r \rightarrow \infty}\left(r^{2} \inf _{|x|=r} \operatorname{Re}(-q(x))\right)=\infty .
$$

Before we state and prove Theorem 5.10, we are going to unify Assumptions 2.1, 4.2, 5.5 and 5.8 in more organized form:

Assumption 5.9. (1) Let $N$ be an integer such that $N \geq 2$. Let $u \in H^{2}\left(E_{R_{0}}\right)_{\text {loc }}$, $R_{0}>0$, be a solution of the homogeneous Schrödinger equation (2.1), where $E_{R_{0}}$ is given by (2.2) (with $R=R_{0}$ ). Here $q(x)$ is a complex-valued, measurable, locally bounded function on $E_{R_{0}}$ which satisfies (5.30).

(2) Set

$$
Q(x)=q(x)+\frac{(N-1)(N-3)}{4 r^{2}} .
$$

(2-a) Then $Q(x)$ is decomposed as

$$
Q(x)=Q_{0}(x)+Q_{1}(x),
$$

where $Q_{0}(x)$ is a non-positive, measurable, locally bounded function on $E_{R_{0}}$ and $Q_{1}(x)$ is a complex-valued, measurable, locally bounded function on $E_{R_{0}}$ such that

$$
0 \geq \beta Q_{0}(x) \geq \operatorname{Re}(Q(x)) \quad\left(x \in E_{R_{0}}\right)
$$

with a constant $\beta \in(0,1)$.

(2-b) For any $\phi \in X=L_{2}\left(S^{N-1}\right),\left(Q_{0}(r \cdot) \phi, \phi\right)$ has the right limit for all $r>R_{0}$ as a function of $r=|x|$, where $($,$) is the inner product of X$.

(2-c) There exist $h_{0}>0$ and, for $0<h<h_{0}$, a real-valued, measurable function $Q_{0 r}(x ; h)$ on $E_{R_{0}}$ such that $(2.6),(2.7)$ and (2.8) hold.

(2-d) There exists $h(r) \in L_{1}\left(\left(R_{0}, \infty\right)\right)$ such that

$$
0<h(r) \leq \frac{2}{r} \quad\left(r>R_{0}\right)
$$

and, setting

$$
\left\{\begin{aligned}
& a(r)=h^{-1}(r) \sup _{|x|=r}\left|Q_{1}(x)\right|, \\
& b(r)=\inf _{|x|=r}\left[-\left(Q_{0}(x)+h^{-1}(r) Q_{0 r}(x)\right)\right], \\
&\left(h^{-1}(r)=1 / h(r)\right),
\end{aligned}\right.
$$


we have

$$
a(r)^{2} \leq b(r) \quad\left(r>R_{0}\right)
$$

(3) The function $F(r)$ introduced in Definition 4.1 satisfies Assumption 4.2.

Theorem 5.10. Suppose that Assumptions 5.9 hold. Suppose that the solution $u$ satisfies (5.1). Then u has compact support.

Proof. Note that

$$
\begin{aligned}
0 \leq\left|v^{\prime}(r)\right|^{2}- & \left(C_{R}(r) v(r), v(r)\right) \\
= & \left|\left(r^{(N-1) / 2} u(r \cdot)\right)^{\prime}\right|^{2}-r^{N-1}(\operatorname{Re}(Q(r \cdot)) u(r \cdot), u(r \cdot)) \\
= & r^{N-1}\left|\partial_{r} u(r \cdot)+2^{-1}(N-1) r^{-1} u(r \cdot)\right|^{2} \\
& -r^{N-1}(\operatorname{Re}(Q(r \cdot)) u(r \cdot), u(r \cdot)) \\
\leq & 2 r^{N-1}\left|\partial_{r} u(r \cdot)\right|^{2}+2^{-1}(N-1)^{2} r^{N-3}|u(r \cdot)|^{2} \\
& -r^{N-1}\left(\left\{\operatorname{Re}(q(r \cdot))+\frac{(N-1)(N-3)}{4 r^{2}}\right\} u(r \cdot), u(r \cdot)\right), \\
\leq & 2 r^{N-1}\left|\partial_{r} u(r \cdot)\right|^{2}+\frac{N^{2}-1}{4} r^{N-3}|u(r \cdot)|^{2} \\
- & r^{N-1}(\operatorname{Re}(q(r \cdot)) u(r \cdot), u(r \cdot)),
\end{aligned}
$$

where $\partial_{r}=\partial / \partial r$ and we have used

$$
\left\{\begin{array}{l}
Q(x)=q(x)+\frac{(N-1)(N-3)}{4 r^{2}} \\
\frac{(N-1)^{2}}{2}-\frac{(N-1)(N-3)}{4}=\frac{N^{2}-1}{4} .
\end{array}\right.
$$

Therefore we have

$$
\begin{aligned}
& 0 \leq\left|v^{\prime}(r)\right|^{2}-\left(C_{R}(r) v(r), v(r)\right) \\
& \leq 2 r^{N-1}\left\{\left|\partial_{r} u(r \cdot)\right|^{2}-(\operatorname{Re}(q(r \cdot)) u(r \cdot), u(r \cdot))\right\} \\
& \quad-r^{N-3}\left(\left[-r^{2} \operatorname{Re}(q(r \cdot))-4^{-1}\left(N^{2}-1\right)\right] u(r \cdot), u(r \cdot)\right) .
\end{aligned}
$$

It follows from (1) of Assumption $5.9((5.30))$ that there exists $R_{3}>R_{0}$ such that

$$
-r^{2} \operatorname{Re}(q(x))-4^{-1}\left(N^{2}-1\right)>0 \quad\left(|x|=r, r \geq R_{3}\right),
$$


and hence, for $|x| \geq R_{3}$,

$$
\begin{aligned}
0 \leq\left|v^{\prime}(r)\right|^{2} & -\left(C_{R}(r) v(r), v(r)\right) \\
& \leq 2 \int_{|x|=r}\left\{\left|\frac{\partial u}{\partial r}\right|^{2}-\operatorname{Re}(q(x))|u|^{2}\right\} d S,
\end{aligned}
$$

which, together with (5.1), implies (5.29). Thus Corollary 5.7 can be applied to see that $u$ has compact support, which completes the proof. 


\section{$\S 6$. Examples}

In this section we are going to give some applications of Theorem 5.10.

Example 6.1. Let $\bar{R}>0$ and let $u \in H^{2}\left(E_{\bar{R}}\right)_{\text {loc }}$ be a solution of the equation

$$
\left(-\Delta+V_{\ell}(x)+V_{s}(x)-\lambda(x)\right) u=0 \quad\left(x \in E_{\bar{R}}\right)
$$

Here $\lambda(x)$ is a real-valued, measurable, locally bounded function on $E_{\bar{R}}$ satisfying the following (i) and (ii):

(i) There exists $m_{0}>0$ such that

$$
\lambda(x) \geq m_{0} \quad\left(x \in E_{\bar{R}}\right) .
$$

(ii) For any $\phi \in X$ the function

$$
f_{\phi}(r)=(\lambda(r \cdot) \phi, \phi)
$$

is a right continuous, nondecreasing function on $(\bar{R}, \infty)$.

The functions $V_{\ell}(x)$ and $V_{s}(x)$ are real-valued and complex-valued functions, respectively, satisfying the following (iii) and (iv):

(iii) The long-range potential $V_{\ell}(x)$ is assumed to be $C^{1}$ function on $E_{\bar{R}}$ such that

$$
\left\{\begin{array}{l}
\lim _{r \rightarrow \infty} \sup _{|x|=r}\left|V_{\ell}(x)\right|=0, \\
\sup _{r>\bar{R},|x|=r}\left\{|x|^{1+\epsilon}\left|\frac{\partial V_{\ell}}{\partial|x|}\right|\right\}<\infty
\end{array}\right.
$$

with $\epsilon \in(0,2)$.

(iv) The short-range potential $V_{s}(x)$ is assumed to be measurable such that

$$
\sup _{r>\bar{R},|x|=r}\left\{r^{1+\epsilon}\left|V_{s}(x)\right|\right\}<\infty
$$

where $\epsilon$ is as above.

Suppose, in addition, that

$$
\lim _{r \rightarrow \infty} \int_{|x|=r}\left\{\left|\frac{\partial u}{\partial r}\right|^{2}+\lambda(x)|u|^{2}\right\} d S=0 .
$$


Then $u$ is identically zero in $E_{\bar{R}}$. In fact, set

$$
\left\{\begin{array}{l}
Q_{0}(x)=-\lambda(x)+V_{\ell}(x) \\
Q_{0 r}(r \omega ; h)=h^{-1} \int_{r}^{r+h} \frac{\partial V_{\ell}}{\partial r}(s \omega) d s \quad\left(\omega \in S^{N-1}, h>0\right), \\
Q_{0 r}(x)=\frac{\partial V_{\ell}}{\partial r} \\
Q_{1}(x)=V_{s}(x)+\frac{(N-1)(N-3)}{4 r^{2}}
\end{array}\right.
$$

Then, since

$$
\operatorname{Re}(-q(x))=\lambda(x)-V_{\ell}(x)-\operatorname{Re}\left(V_{s}(x)\right) \geq m_{0}-V_{\ell}(x)-\operatorname{Re}\left(V_{s}(x)\right)
$$

(1) of Assumption 5.9 is satisfied for sufficiently large $r$. For $\phi \in X$, we have

$$
\begin{aligned}
\frac{1}{h}\left(\left[Q_{0}((r\right.\right. & \left.\left.+h) \cdot)-Q_{0}(r \cdot)\right] \phi, \phi\right) \\
& =-\frac{1}{h}([\lambda((r+h) \cdot)-\lambda(r \cdot)] \phi, \phi)+\frac{1}{h}\left(\left[V_{\ell}((r+h) \cdot)-V_{\ell}(r \cdot)\right] \phi, \phi\right) \\
& \leq\left(Q_{0 r}(r \cdot ; h) \phi, \phi\right) \\
& \rightarrow\left(Q_{0 r}(r \cdot) \phi, \phi\right)
\end{aligned}
$$

as $h \rightarrow 0$ with $h>0$. Thus (2-c) of Assumption 5.9 is satisfied. Set

$$
h(r)=r^{-1-\epsilon / 2} \quad(r>\bar{R}) .
$$

Then $h(r) \in L_{1}((\bar{R}, \infty))$ and the inequality (5.34) is satisfied for sufficiently large $r$. Also we have $a(r) \rightarrow 0$ as $r \rightarrow \infty$ and $b(r) \geq m_{0} / 2$ for sufficiently large $r$, and hence (2-d) of Assumption 5.9 is now satisfied. Noting that

$$
\beta Q_{0}(x)-\operatorname{Re}(Q(x))=(1-\beta) \lambda(x)+(\beta-1) V_{\ell}(x)-\operatorname{Re}\left(V_{s}(x)\right)
$$

and that $\lambda(x) \geq m_{0}((6.2))$, we see that (2-a) of Assumption 5.9 holds for sufficiently large $r$ with any $\beta \in(0,1)$. The condition (2-b) of Assumption 5.9 is verified by (ii) of Example 6.1 and the smoothness of $V_{\ell}(x)$. Define $F(r)$ by $F(r)=\log r$. Obviously (ii) and (iii) of Assumption 4.2 are satisfied by definition. Since

$$
r^{4} h^{2}(r) b(r)=r^{2-\epsilon}(\lambda(x)+o(1))
$$


as $r \rightarrow \infty$, (4.5) in Assumption 4.2 holds for sufficiently large $r$. Therefore, by setting $R_{0}$ sufficiently large, all the conditions of Assumption 5.9 are satisfied, which implies that the solution $u$ has compact support in $E_{\bar{R}}$. Therefore it follows from the unique continuation theorem that $u$ is identically zero in $E_{\bar{R}}$.

We remark here that, if $\lambda(x)$ is assumed to be bounded from above, too, then the condition (6.6) is equivalent to

$$
\varliminf_{r \rightarrow \infty} \int_{|x|=r}\left\{\left|\frac{\partial u}{\partial r}\right|^{2}+|u|^{2}\right\} d S=0
$$

Another remark is that, if $V_{s}(x)$ is real-valued, then the condition (6.6) is implied by the generalized radiation condition

$$
r^{\delta-1}\left(\frac{\partial u}{\partial r}-i \sqrt{\lambda(x)} u\right) \in L_{2}\left(E_{R}\right)
$$

with $\delta>1 / 2$ and $R>\bar{R}$.

Example 6.2. Let $\bar{R}>0$ and let $u \in H^{2}\left(E_{\bar{R}}\right)_{\text {loc }}$ be a solution of the equation

$$
\left(-\frac{1}{\mu(x)} \Delta-\lambda\right) u=0 \quad\left(x \in E_{\bar{R}}\right)
$$

Here $\lambda>0$ and the real-valued function $\mu(x)$ on $E_{\bar{R}}$ is decomposed as

$$
\mu(x)=\mu_{0}(x)+\mu_{\ell}(x)+\mu_{s}(x) \quad\left(x \in E_{\bar{R}}\right),
$$

where $\mu_{0}(x), \mu_{\ell}(x)$ and $\mu_{s}(x)$ satisfy the following $(\mathrm{i}) \sim(\mathrm{iv})$ :

(i) $\mu_{0}(x)$ is real-valued and measurable and there exists $\widetilde{m_{0}}>0$ such that

$$
\mu_{0}(x) \geq \widetilde{m_{0}} \quad\left(x \in E_{\bar{R}}\right) .
$$

(ii) For any $\phi \in X$ the function

$$
g_{\phi}(r)=\left(\mu_{0}(r \cdot) \phi, \phi\right)
$$

is a right continuous, nondecreasing function on $(\bar{R}, \infty)$.

(iii) The real-valued function $\mu_{\ell}$ satisfies Example 6.1, (iii) with $V_{\ell}(x)$ replaced by $\mu_{\ell}$.

(iv) The complex-valued function $\mu_{s}(x)$ satisfies Example 6.1, (iv) with $V_{s}(x)$ replaced by $\mu_{s}$. 
Set

$$
\left\{\begin{array}{l}
\lambda(x)=\lambda \mu_{0}(x), \\
V_{\ell}(x)=\lambda \mu_{\ell}(x), \\
V_{s}(x)=\lambda \mu_{s}(x)
\end{array}\right.
$$

Then $u$ satisfy the equation (6.1) in Example 6.1, where $\lambda(x), V_{\ell}(x)$ and $V_{s}(x)$ satisfy (i) (iv) in Example 6.1. Thus the condition

$$
\lim _{r \rightarrow \infty} \int_{|x|=r}\left\{\left|\frac{\partial u}{\partial r}\right|^{2}+\lambda \mu_{0}(x)|u|^{2}\right\} d S=0
$$

implies that $u$ is identically zero.

\section{$\S 7$ Reduced Wave operator in layered media}

In [8] we considered the reduced wave operator

$$
H=-\frac{1}{\mu(x)} \Delta \quad \text { in } \mathcal{H}=L_{2}\left(\mathbf{R}^{N}, \mu(x) d x\right),
$$

where $\mu(x)$ is a real-valued function such that

$$
0<\inf _{x} \mu(x) \leq \sup _{x} \mu(x)<\infty .
$$

By defining the domain $D(H)$ of $H$ by $D(H)=H^{2}\left(\mathbf{R}^{N}\right)$, where $H^{2}\left(\mathbf{R}^{N}\right)$ is the second order Sobolev space on $\mathbf{R}^{N}, H$ becomes a self-adjoint operator on $\mathcal{H}$. In this section we shall show that the nonexistence of the eigenvalues of $H$ can be proved in some cases discussed in [8] by using the result of $\S 6$ (Example 6.2). Suppose that $\mu(x)$ has the decomposition (6.16) with a positive function $\mu_{0}$, a long-range perturbation $\mu_{\ell}$ and a short-range perturbation $\mu_{s}$. The functions $\mu_{0}, \mu_{\ell}$ and $\mu_{s}$ are assumed to satisfy (i) (iv) of Example 6.2. In [8], for the sake of simplicity, we assumed that only one of a long-range perturbation or short-range perturbation appeared with the main term $\mu_{0}(x)$, but we can easily modify the arguments in [8] so that we can treat $\mu(x)$ of the form (6.16). Let $K_{-}$be a nonpositive integer or $K_{-}=-\infty$ and let $K_{+}$be a nonnegative integer or $K_{+}=\infty$. Let $K$ be a set of integers given by

$$
K=\left\{k / K_{-} \leq k \leq K_{+}\right\} .
$$


Let $\left\{\Omega_{k}\right\}_{k \in K}$ be a sequence of open sets of $\mathbf{R}^{N}$ such that

$$
\left\{\begin{array}{l}
\Omega_{k} \cap \Omega_{\ell}=\emptyset \quad(k \neq \ell), \\
\bigcup_{k \in K} \overline{\Omega_{k}}=\mathbf{R}^{N},
\end{array}\right.
$$

where $\bar{A}$ is the closure of $A$. Further we assume that the boundary $\partial \Omega_{k}$ of $\Omega_{k}$ has the form

$$
\partial \Omega_{k}=S_{k}^{(-)} \cup S_{k}^{(+)}
$$

where $S_{k}^{(-)} \cap S_{k}^{(+)}=\emptyset$, and each of $S_{k}^{(-)}$and $S_{k}^{(+)}$is a continuous surface which is a finite union of smooth surfaces. We also assume that

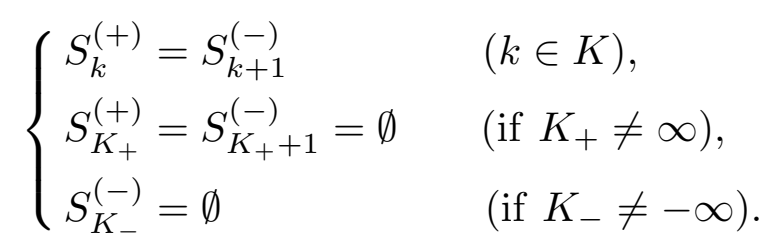

Now the function $\mu_{0}(x)$ is assumed to be a simple function which takes a constant value $\nu_{k}$ on each $\Omega_{k}$ such that $\left\{\nu_{k}\right\}_{k \in K}$ ia a bounded, positive sequence. We assume that the origin 0 of the coordinates is in $\Omega_{0}$, and $\mu_{0}(x)$ satisfies the condition

$$
\left(\nu_{k+1}-\nu_{k}\right)\left(n^{(k)}(x) \cdot x\right) \geq 0 \quad\left(x \in S_{k}^{(+)}=S_{k+1}^{(-)}, k \in K\right)
$$

where $n^{(k)}(x)$ is the unit outward normal of $\Omega_{k}$ at $x \in \partial \Omega_{k}$ and $n^{(k)}(x) \cdot x$ is the inner product of $n^{(k)}(x)$ and $x$ in $\mathbf{R}^{N}$. Then the following theorem has been obtained in [8] ([8], Theorem 4.6):

Theorem 7.1. Let $H$ be as above. Suppose, in addition, that $\mu$ takes the form of either $\mu=\mu_{0}+\mu_{s}$ or $\mu=\mu_{0}+\mu_{\ell}$. Let $\sigma_{p}(H)$ be the set of the point spectrum of $H$. Then the multiplicity of each $\lambda \in \sigma_{p}(H)$ is finite, $\sigma_{p}(H)$ does not have any accumulation points except at 0 and $\infty$.

It is not difficult to extend this result to the general case that $\mu=\mu_{0}+\mu_{s}+\mu_{\ell}$. Using the Example 6.2, we can show a sufficient condition for the nonexistence of the point spectrum of the operator $H$.

Theorem 7.2. Let $H$ be as above. Suppose that, for almost all $\omega \in S^{N-1}$, $\mu_{0}(r \omega)$ is a nondecreasing function of $r \in[0, \infty)$. Then $\sigma_{p}(H)=0$. 
Proof. The condition (ii) of Example 6.2 is now satisfied since $\mu_{0}(r \omega)$ is nondecreasing.

Here we are going to give some examples.

Example 7.3. Let $\left\{U_{k}\right\}_{k=0}^{\infty}$ be a sequence of open sets of $\mathbf{R}^{N}$ such that

$$
\left\{\begin{array}{l}
\overline{U_{k}} \subset U_{k+1} \quad(k \geq 0), \\
\bigcup_{k=0}^{\infty} U_{k}=\mathbf{R}^{N}
\end{array}\right.
$$

where the boundary $\partial U_{k}$ of $U_{k}$ is a continuous surface which is a finite union of smooth surfaces. Suppose that

$$
\tilde{n}^{(k)}(x) \cdot x \geq 0 \quad(k=0,1,2, \cdots),
$$

where $\tilde{n}^{(k)}(x)$ is the unit outward normal of $U_{k}$ at $x \in \partial U_{k}$. Set

$$
\begin{cases}\Omega_{0}=U_{0} & \\ \Omega_{k}=U_{k} \backslash U_{k-1} & (k \geq 1), \\ S_{k}^{(+)}=\partial U_{k} & (k \geq 0) \\ S_{k}^{(-)}=\partial U_{k-1} & (k \geq 1) .\end{cases}
$$

This is the case that $K_{-}=0$ and $K_{+}=\infty$. Let $\mu_{0}(x)$ be given by

$$
\mu_{0}(x)=\nu_{k} \quad\left(x \in \Omega_{k}\right),
$$

where $\left\{\nu_{k}\right\}_{k=0}^{\infty}$ ia a bounded, positive, increasing sequence. Then we see that not only the condition (7.7) is satisfied but also $\mu_{0}(r \omega)$ is a nondecreasing function of $r \in[0, \infty)$ for almost all $\omega \in S^{N-1}$. Thus Theorem 7.2 can be applied to see that there is no point spectrum of $H$. Therefore the limitig absorption principle holds on the whole positive interval $(0, \infty)$ (see $\S 5$ of $[8]$ ).

Example 7.4. Let $\left\{c_{k} / k= \pm 1, \pm 2, \cdots\right\}$ be an increasing sequence of real numbers such that

$$
\left\{\begin{array}{l}
c_{-1}<0<c_{1} \\
\lim _{k \rightarrow \pm \infty} c_{k}= \pm \infty .
\end{array}\right.
$$


Let $x_{N}$ be the N-th coordinate of $x=\left(x_{1}, x_{2}, \cdots, x_{N}\right)$, and set

$$
\Omega_{k}= \begin{cases}\left\{x \in \mathbf{R}^{N} / c_{-1}<x_{N}<c_{1}\right\} & (k=0), \\ \left\{x \in \mathbf{R}^{N} / c_{k-1}<x_{N}<c_{k}\right\} & (k=-1,-2, \cdots), \\ \left\{x \in \mathbf{R}^{N} / c_{k}<x_{N}<c_{k+1}\right\} & (k=1,2, \cdots) .\end{cases}
$$

We also set

$$
\begin{aligned}
& S_{0}^{( \pm)}=\left\{x \in \mathbf{R}^{N} / x_{N}=c_{ \pm 1}\right\}, \\
& S_{k}^{(+)}= \begin{cases}\left\{x \in \mathbf{R}^{N} / x_{N}=c_{k}\right\} & (k=-1,-2, \cdots), \\
\left\{x \in \mathbf{R}^{N} / x_{N}=c_{k+1}\right\} & (k=1,2, \cdots),\end{cases}
\end{aligned}
$$

and

$$
S_{k}^{(-)}= \begin{cases}\left\{x \in \mathbf{R}^{N} / x_{N}=c_{k-1}\right\} & (k=-1,-2, \cdots), \\ \left\{x \in \mathbf{R}^{N} / x_{N}=c_{k}\right\} & (k=1,2, \cdots),\end{cases}
$$

Note that, for $x \in S_{k}^{(+)}$,

$$
n^{(k)}(x) \cdot x \begin{cases}\geq 0 & (k \geq 0) \\ \leq 0 & (k<0) .\end{cases}
$$

Define a simple function $\mu_{0}(x)$ by $(7.11)$, where the sequence $\left\{\nu_{k}\right\}_{k=-\infty}^{\infty}$ is assumed to be bounded and positive such that $\left\{\nu_{k}\right\}_{k=-\infty}^{-1}$ is decreasing and $\left\{\nu_{k}\right\}_{k=1}^{\infty}$ is increasing. Then, as in Example 7.3, Theorem 7.2 can be applied to show that $\sigma_{p}(H)=\emptyset$. The planes $\left\{x \in \mathbf{R}^{N} / x_{N}=c_{k}\right\}$ can be perturbed as far as the condition (7.17) is satisfied.

\section{Appendix}

Here we are going to prove a lemma on distributions on a half interval $(a, \infty)$ which was used when we evaluate the functionals $M^{+}(v, r)$ and $N(v, m, r)$.

Lemma A. Let $f(r)$ be a real-valued function on $I=(a, \infty)$ such that $f$ is locally $L_{1}$ and right continuous on $I$. Suppose that $f^{\prime} \geq 0$, where $f^{\prime}$ is the distributional derivative of $f$ and the inequality should be taken in the sense of distributions. Then $f$ is nondecreasing on $I$. 
Proof. Here we are giving a rather elementary proof.

(I) Let $r \in I$ and $h>0$. Then, for $\phi \in C_{0}^{\infty}(I)$, we have

$$
\begin{aligned}
\int_{I} & {[f(r+h)-f(r)] \phi(r) d r } \\
& =-\int_{I} f(r)[\phi(r)-\phi(r-h)] d r \\
& =-\int_{I} f(r) \int_{r-h}^{r} \phi^{\prime}(s) d s d r
\end{aligned}
$$

where $\phi$ is supposed to be extended on the whole line $(-\infty, \infty)$ by setting $\phi(r)=$ 0 for $r \leq a$. Since

$$
\int_{r-h}^{r} \phi^{\prime}(s) d s=\int_{0}^{h} \phi^{\prime}(t+r-h) d t
$$

it follows that

$$
\int_{I}[f(r+h)-f(r)] \phi(r) d r=\int_{0}^{h}\left[-\int_{I} f(r) \phi^{\prime}(t+r-h) d r\right] d t .
$$

(II) Let $\phi \in C_{0}^{\infty}(I)$ and $\phi \geq 0$. Then, since

$$
-\int_{I} f(r) \phi^{\prime}(t+r-h) d r=<f^{\prime}, \phi(\cdot+t-h)>\geq 0
$$

for $h>0$ and $0 \leq t \leq h$, where $<F, G>$ denotes the value of the distribution $F$ for the test function $G$, it follows from (A.3) that

$$
\int_{I}[f(r+h)-f(r)] \phi(r) d r \geq 0
$$

for any $\phi \in C_{0}^{\infty}(I)$ with $\phi \geq 0$.

(III) Suppose that there exist $r_{0} \in I, h_{0}>0$ and $\eta_{0}>0$ such that

$$
f\left(r_{0}+h_{0}\right)-f\left(r_{0}\right)=-\eta_{0} .
$$

Since $f$ is right continuous, there exists $r_{1}>r_{0}$ such that

$$
\left\{\begin{array}{l}
\left|f\left(r_{0}\right)-f(r)\right|<\eta_{0} / 3, \\
\left|f\left(r_{0}+h_{0}\right)-f\left(r+h_{0}\right)\right|<\eta_{0} / 3
\end{array}\right.
$$


for $r_{0} \leq r \leq r_{1}$. Then, for $r_{0} \leq r \leq r_{1}$, we have

$$
\begin{aligned}
f\left(r+h_{0}\right) & -f(r) \\
& =f\left(r_{0}+h_{0}\right)-f\left(r_{0}\right)+\left\{f\left(r+h_{0}\right)-f\left(r_{0}+h_{0}\right)\right\}+\left\{f\left(r_{0}\right)-f(r)\right\} \\
& \leq f\left(r_{0}+h_{0}\right)-f\left(r_{0}\right)+\left|f\left(r+h_{0}\right)-f\left(r_{0}+h_{0}\right)\right|+\left|f\left(r_{0}\right)-f(r)\right| \\
& <-\eta_{0} / 3 .
\end{aligned}
$$

Let $\phi \in C_{0}^{\infty}(I)$ such that

$$
\left\{\begin{array}{l}
\operatorname{supp} \phi \subset\left[r_{0}, r_{1}\right] \\
\phi \geq 0 \\
\int_{r_{0}}^{r_{1}} \phi(r) d r=1 .
\end{array}\right.
$$

Then, it follows that

$$
\begin{aligned}
\int_{I}[f(r+h)-f(r)] \phi(r) d r & =\int_{r_{0}}^{r_{1}}[f(r+h)-f(r)] \phi(r) d r \\
& \leq-\frac{\eta_{0}}{3} \int_{r_{0}}^{r_{1}} \phi(r) d r \\
& =-\frac{\eta_{0}}{3}<0
\end{aligned}
$$

which contradicts (A.5). This completes the proof.

\section{Refenreces}

[1] D. Eidus, The principle of limiting absorption, American Math. Soc. Translations 47 (1965), 157-191 (Mat. Sb. 57 (1962)).

[2] D. Eidus, The limiting absorption and amplitude problems for the diffraction problem with two unbounded media, Comm. Math. Phys. 107 (1986), 29-38.

[3] T. Ikebe, Eigenfunction expansions associated with the Schrödinger operators and their applications to scattering theory, Arch. Rational Mech. Anal. 5 (1960), 1-34.

[4] T. Ikebe and Y. Saitō, Limiting absorption method and absolute continuity for the Schrödinger operator, J. Math. Kyoto Univ. 12 (1972), 513-612..

[5] T. Ikebe and J. Uchiyama, On the asymptotic behavior of eigenfunctions of secondorder elliptic differential operators, J. Math. Kyoto Univ. 11 (1971), 425-448. 
[6] W. Jäger, Zur Theorie der Schwingugsgleichung mit variablen Koeffizienten in Aussengebieten, Math. Z. 102 (1969), 62-88.

[7] W. Jäger and Y. Saitō, On the Spectrum of the Reduced Wave Operator with Cylindrical Discontinuity, Forum Mathematicum 9 (1997), 29-60.

[8] W. Jäger and Y. Saitō, The reduced wave equation in layered materials, to appear in Osaka J. Math..

[9] T. Kato, Growth properties of solutions of the reduced wave equation with a variable coefficients, Comm. Pure Appl. Math. 12 (1959), 403-425.

[10] C. Müller, Grundprobleme der mathematischen Theorie elektromagnetischer Schwingungen, Springer, Berlin, 1957.

[11] F. Rellich, Über das asymptotische Verhalten des Lösungen von $\Delta u+\lambda u=0$, Jber. Deutsche. Math. Verein. 53 (1943), 57-65.

[12] G. Roach and B. Zhang, On Sommerfeld radiation conditions for the diffraction problem with two unbounded media, Proc. Royal Soc. Edinburgh 121A (1992), 149-161.

[13] Y. Saitō, Spectral Representations for Schrödinger Operators with Long-Range Potentials, Lecture Notes in Mathematics, vol. 727, Springer, Berlin, 1979.

[14] J. Weidmann, On the Continuous spectrum of Schrödinger operators, Comm. Pure and Appl. Math. 19 (1966), 107-110.

[15] E. Wienholtz, Halbbeschränke partielle Differentialoperatoren zweiter Ordnung vom elliptischen Typus, Math. Ann. 135 (1958), 50-80. 